\title{
EFFECT OF PIOGLITAZONE ON THE BONE HEALTH OF DIABETIC PATIENTS IN JEDDAH, KSA
}

\author{
Abdulrahman M. Alahdal, Mahmoud A. Abdel-Hameed, Alaa Alghamdi, Doha \\ Alamoudi, Khulood Almehmadi, Rana Alhashmi \\ Department of Clinical Pharmacy, Faculty of Pharmacy, KAU
}

\begin{abstract}
Background: Osteoporosis is an important health care issue because the resultant bone fractures cause great deal of morbidity and mortality. Among the important risk factors for the development of osteoporosis is diabetes mellitus.

Objective: Thiazolidinediones (TZDs), group of antidiabetic drugs used to reduce insulin resistance, have been inconsistently shown to be associated with osteoporotic fractures. The purpose of this study was to find out if the use of pioglitazone, an oral antidiabetic medication belonging to TZDs, has any adverse effects on bone health in patients with type- 2 diabetes in Jeddah, KSA.
\end{abstract}

Methods: This case control analysis included 56 subjects, 40 to 65 years old (18 diabetic patients under pioglitazone therapy, 18 diabetic patients under metformin-sulfonylurea therapy, and 20 non-diabetic persons) enrolled in osteoporosis center in Jeddah, KSA. Medication information was taken directly from drug containers during in-person interviews. The main outcome measures were the bone mineral density (BMD) and the serum levels of vitamin D, calcium, and creatinine.

Results: No significant BMD and biochemical (serum vitamin D, calcium, and creatinine) changes were be detected in the three groups (Pioglitazone users group; metformin-sulfonylurea users group; and the nondiabetic persons group).

Conclusion: Pioglitazone does not cause adverse effect on the BMD and biochemical tests. This is in contrast to other studies that showed decreased BMD and increased risk of fracture in patients on TZDs. Some of this contravention may be explained by factors that are included within bone quality, while some may be related to the other non-skeletal factors. In terms of bone quality, there have been suggestions that there are racial differences in bone strength due to genetic, nutritional, lifestyle, and hormonal factors. The possible other non-skeletal factors include the small number of patients studied in the present work as well as the variation of the dose of the drug, and the duration of therapy. Therefore, large prospective, randomized trials are necessary to evaluate the effect of pioglitazone on the bone health of diabetic patients in KSA.

\section{INTRODUCTION AND AIM}

The insisting importance of therapy of diabetes mellitus is emphasized by the fact that the disease affects a large number of populations and causes many disabling complications. Although traditionally osteoporosis has not been listed as diabetes related complication, increasing evidence suggests that patients with type 1 or type 2 diabetes are at increasing risk of osteoporotic fracture (Brandi, 2010). Therefore, it was proposed that hyperglycemia, a result of insulin deficiency or insulin resistance, is responsible for higher osteoporosis in the population with diabetes (Brandi, 2010). Higher glucose levels in the blood are known to interact with 
several proteins to form advanced glycation end (AGE) products that may interact with bone to reduce bone strength, resulting in osteoporosis in patients with diabetes (Yamagishi, et al., 2005; Alikiani, et al., 2007; Munoz, et al., 1996). Another indirect effect of hyperglycemia is glycosuria, which causes hypercalciuria, leading to decreased levels of calcium in the body and poor bone quality. Additionally, several diabetes related complications such as retinopathy, neuropathy, and nephropathy have been linked to decreased BMD (Monami et al., 2008).

Thiazolidinediones (TZDs) are class of oral hypoglycemic drugs that have been commonly prescribed throughout the world for the treatment of type 2 diabetes mellitus both as monotherapy and in combination with metformin, sulfonylurea and insulin (Wannakamo, et al., 2012; Priya and Sunder, 2010). Recent evidence from an epidemiological study and an exploratory clinical trial suggests that the use of the insulin-sensitizing agents, TZDs is associated with a decrease in bone density in postmenopausal women (Schwartz, et al., 2006; Grey, et al., 2007). Similar results have been reported by an observational retrospective study exploring variations of bone density in older men with type 2 diabetes (Yaturu, et al., 2007). In vitro and in vivo studies demonstrated that peroxisome proliferator-activated receptor induction in mesenchymal cells leads to increased adipogenesis and decreased osteoblastogenesis (Canalis, et al., 2007). Thiazolidinediones also decrease the expression of insulin-like growth factor I, and this may contribute to decreased bone formation (Giustina, et al., 2008). In addition, thiazolidinediones promote osteoclast differentiation and bone resorption (Wan, et al., 2007; Okazaki, et al., 1999). On the other hand, the insulin-sensitizing effect of TZDs reduces circulating insulin levels and therefore the insulin anabolic effect on the bone is reduced (Thrailkill, et al., 2005).

The purpose of this study was to find out if the use of pioglitazone, an oral antidiabetic drug belonging to TZDs class of medications, has any adverse effects on BMD and serum levels of vitamin D, calcium, and creatinine in diabetic patients in Jeddah, KSA.

\section{PATIENTS AND METHODS}

\section{Study Population}

The protocol of this case control study was approved by the ethics committee at the Center of Excellence for Osteoporosis Research (CEOR) at King Abdulaziz University (KAU) in Jeddah, Saudi Arabia. 96 subjects were enrolled between November 2011 and November 2012 at the clinic of CEOR. Participants were recruited randomly from King Abdul-Aziz University Hospital (KAUH), and primary healthcare centers in Jeddah. Out of the total number of 96 participants, 40 were excluded from the study because they did not sign the consent form. Figure 1 shows the study process. Fifty six participants were included in the study and divided into three groups, pioglitazone users $(n=18)$, metformin and sulfonylureas users $(n=18)$, and the control group who were healthy non-diabetic $(n=20)$. All participants were between 40 and 65 years of age, ambulatory, non-alcoholic. Figure 2 shows participants disposition in the study.

Studying the participants characteristics showed that there were no significant differences in age $(\mathrm{p}=0.261)$, gender $(\mathrm{P}=0.298)$, and body mass index (BMI) $(\mathrm{p}=0.187)$ between the three groups, as shown in Table 1.

Other parameters where collected during the study, which was assessed by questionnaires were the sun exposure, exercise and smoking, it showed no significant 
difference between the three groups with a $\mathrm{P}$ value of $(P=0.382)(P=0.430)$ and $(P=0.325)$, respectively, as shown in Table 1.

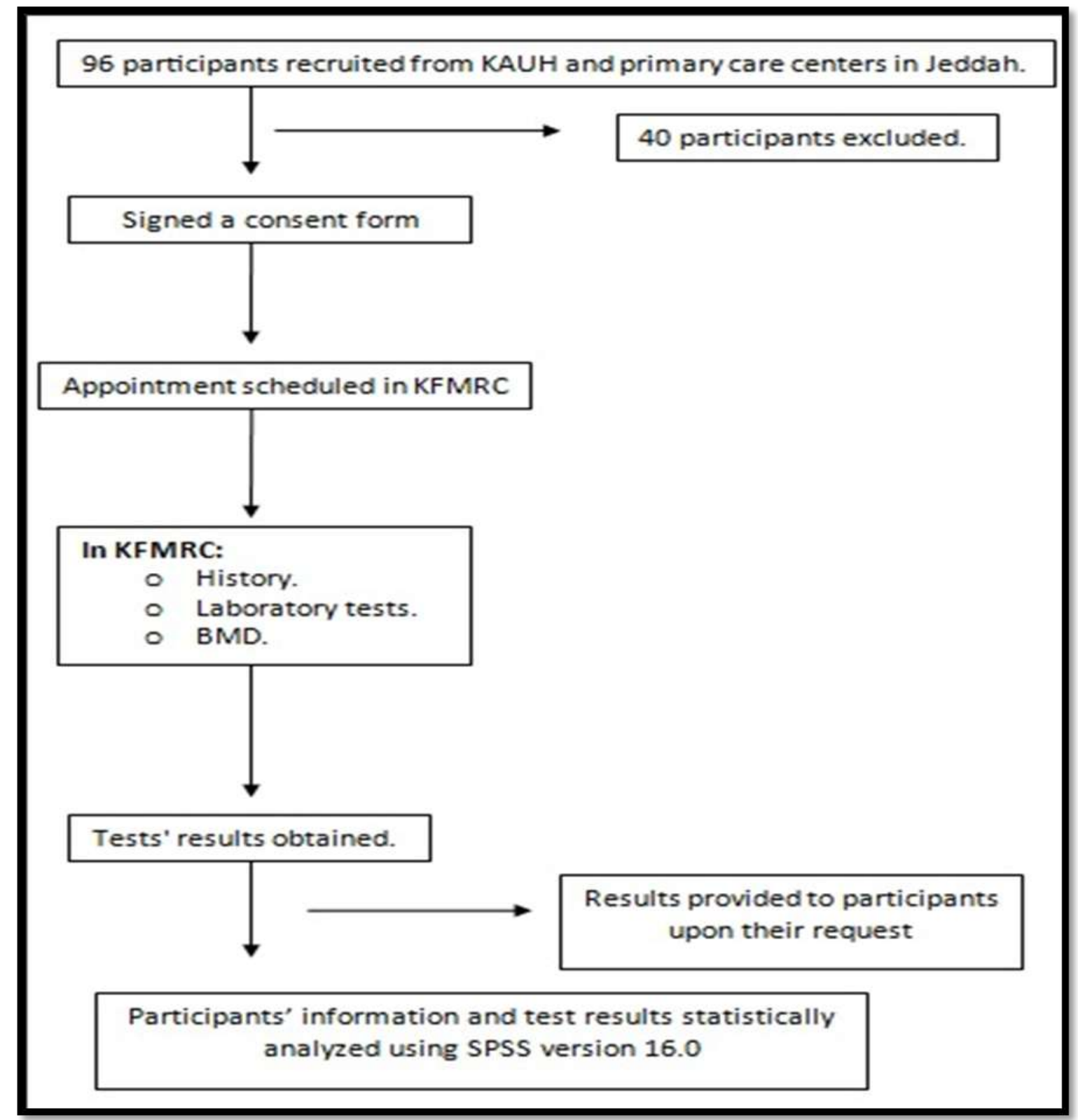

Figure1: study process. KAUH: King Abdul Aziz University Hospital, KFMRC: King Fahd Medical Research Center, BMD: Bone mineral density, DEXA: Dual-Energy X-ray Absorptiometry. 


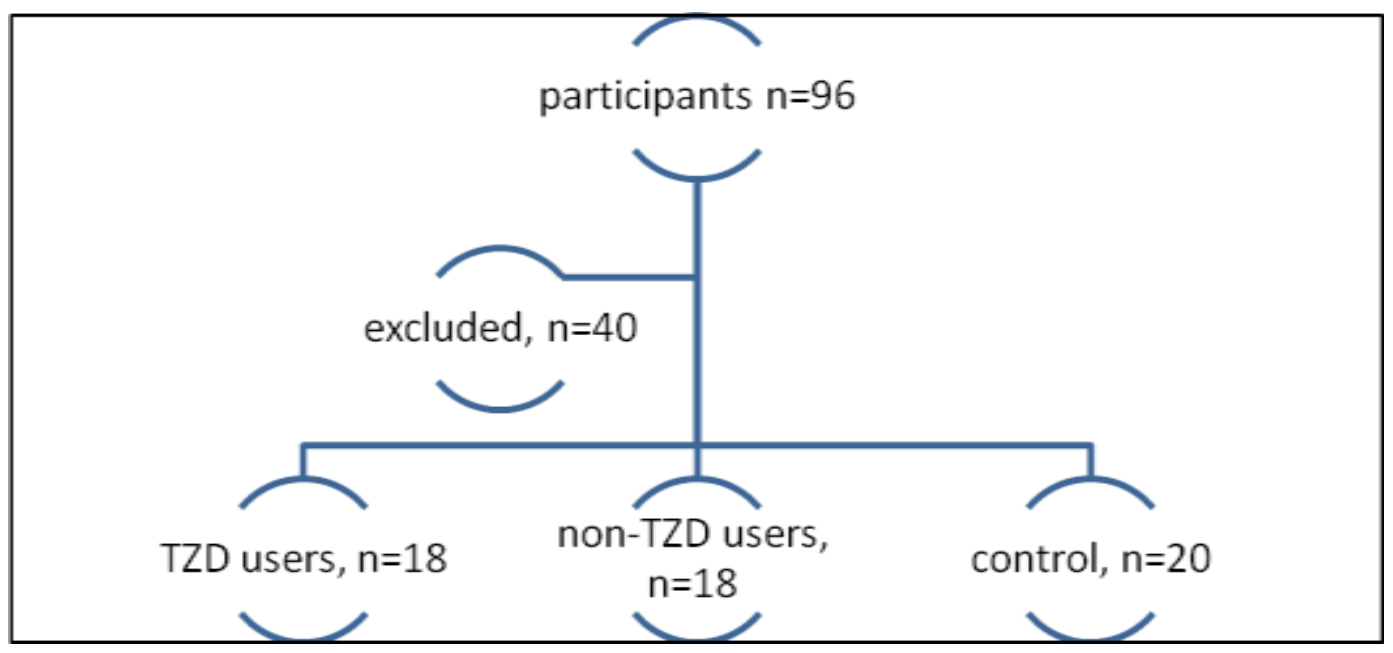

Figure 2: Participants disposition in the study

Table1: characteristics of study subjects.

\begin{tabular}{|l|l|l|l|l|}
\hline Variable & $\begin{array}{l}\text { TZD } \\
\text { users }(\mathrm{n}=18)\end{array}$ & $\begin{array}{l}\text { Non TZD } \\
\text { users(n=18) }\end{array}$ & control $(\mathrm{n}=20)$ & P value \\
\hline Age(year)* & $53.5 \pm 5.2$ & $51.9 \pm 6.3$ & $52 \pm 3.77$ & 0.261 \\
\hline Gender* & $1.8 \pm 0.38$ & $1.6 \pm 0.5$ & $1.65 \pm 0.48$ & 0.298 \\
\hline BMI (Kg/m2)** & $33.7 \pm 5$ & $31.9 \pm 7$ & $30.4 \pm 4$ & 0.187 \\
\hline Sun exposure* & $1.88 \pm 0.832$ & $1.83 \pm 0.7$ & $1.8 \pm 0.58$ & 0.382 \\
\hline Exercise * & $1.38 \pm 0.5$ & $1.5 \pm 0.5$ & $1.6 \pm 0.5$ & 0.430 \\
\hline Smoking* & $1.83 \pm 0.38$ & $1.6 \pm 0.42$ & $1.8 \pm 0.36$ & 0.325 \\
\hline
\end{tabular}

$\mathrm{P}<0.05$ is considered significant.

* $\mathrm{P}$ value calculated by chi-square test.

**P value calculated by one way ANOVA test.

\section{Outcome Ascertainment}

The primary outcome of this study is to examine the BMD by dual-energy X-ray absorptiometry (DXA). Self-reported of previous clinical fractures were collected by questionnaires.

\section{Measurement of BMD}

Body weight was measured with subjects wearing light clothes. Bone mineral density (BMD) at the lumbar spine 2-4 (L2-L4) and the femur were measured by dual-energy X-ray absorptiometry (DXA) (Lunar Corp., USA) by a single experienced technician at one clinical center among 96 subjects. Patients were asked to lie down on their back and by moving the DEXA device on all over the body for one hour the result of the DEXA will be printed.

\section{Serum Tests}

Participants were asked to fast for 10 hours before blood sampling. Four milliliters of blood was withdrawn and collected in coagulated tube (CAT). Serum was prepared by centrifugation at a rate of $1000 \mathrm{x} g(1957 \mathrm{rpm})$ for 10 minutes. Serum tests were done using an analyzer (VITROS ${ }^{8} 250$ Chemistry System by Ortho clinical Diagnostics). Serum 25hydroxyvitamin $\mathrm{D}$ was measured by direct competitive chemiluminescence. Tests requested are (serum Calcium, serum creatinine, and serum vitamin D). 


\section{Statistical Analysis}

The characteristics of patients in the three groups were compared by Chi-square test (for categorical variables) or ANOVA test (for continuous variables). All analyses were conducted using SPSS statistical software version 16.0 (SPSS, Inc., Chicago, Illinois). Statistical significance was set at $\mathrm{P}<0.05$. Data were presented as mean $\pm \mathrm{SD}$.

\section{RESULTS}

\section{BMD results}

There is no significant effect on bone mineral density (BMD) in the three groups. Regarding BMD at the lumbar spine (L2-L4), and femoral neck, BMD measurements at the lumbar spine showed no significant differences between the three groups $(P=0.761)$ with a mean value of $0.91 \pm 0.12,0.95 \pm 0.12$ and $0.94 \pm 0.16$ for TZD users, other ant-diabetic users and the control groups respectively, as shown in Table 2. Similarly, the BMD at the right and left femoral neck also showed no statistical significance with a $\mathrm{P}$ value of $(P=0.959)$ and $(P=$ 0.768) respectively. Where the mean of the BMD was compared using the ANOVA, it showed $0.93 \pm 0.1,0.94 \pm 0.13$ and $0.93 \pm 0.15$ in the three groups, as shown in Table 2 .

\section{Biochemical results}

There were no significant effect on biochemical parameters (vitamin D, calcium and creatinine clearance) in each group and no significant differences in serum vitamin $\mathrm{D}(\mathrm{p}=0.330)$, calcium $(\mathrm{p}=0.455)$, and creatinine clearance $(\mathrm{p}=0.155)$ between the three groups, as shown in Table3.

Table 2: BMD in the Pioglitazone users, Non TZD users, and Non-diabetic groups.

\begin{tabular}{|l|l|l|l|l|}
\hline BMD (g/cm2) & $\begin{array}{l}\text { Pioglitazone } \\
\text { users }(n=18)\end{array}$ & $\begin{array}{l}\text { Non TZD users } \\
(\mathrm{n}=18)\end{array}$ & $\begin{array}{l}\text { Non-diabetics } \\
(\mathrm{n}=20)\end{array}$ & P value \\
\hline L2-L4 & $0.91 \pm 0.12$ & $0.95 \pm 0.12$ & $0.94 \pm 0.16$ & 0.761 \\
\hline Femoral neck (right) & $0.93 \pm 0.1$ & $0.94 \pm 0.13$ & $0.93 \pm 0.15$ & 0.959 \\
\hline Femoral neck (left) & $0.91 \pm 0.12$ & $0.95 \pm 0.12$ & $0.94 \pm 0.16$ & 0.768 \\
\hline
\end{tabular}

- $\mathrm{P}<0.05$ is considered significant value calculated by one way ANOVA test.

- Non-TZD users=Diabetic patients under metformin-sulfonylurea therapy

Table 3: Biochemical results in the Pioglitazone users, Non TZD users, and Non-diabetic groups.

\begin{tabular}{|l|l|l|l|l|}
\hline Variable & $\begin{array}{l}\text { Pioglitazone } \\
\text { users }(\mathrm{n}=18)\end{array}$ & $\begin{array}{l}\text { Non TZD users } \\
(\mathrm{n}=18)\end{array}$ & $\begin{array}{l}\text { Non-diabetics } \\
(\mathrm{n}=20)\end{array}$ & P value \\
\hline $\mathrm{CrCl}(\mathrm{ml} / \mathrm{min})$ & $77.7 \pm 35$ & $100.8 \pm 50.6$ & $94.5 \pm 17.57$ & 0.155 \\
\hline $\begin{array}{l}\text { Serum } \\
\text { calcium(mmol/L) }\end{array}$ & $2.37 \pm 0.08$ & $2.33 \pm 0.1$ & $2.34 \pm 0.1$ & 0.455 \\
\hline $\begin{array}{l}\text { Serum total 25(OH)D } \\
\text { level (nmol/L) }\end{array}$ & $28.9 \pm 13$ & $28.8 \pm 17$ & $36.3 \pm 21$ & 0.330 \\
\hline
\end{tabular}

- $\mathrm{P}<0.05$ is considered significant. ( $\mathrm{P}$ value calculated by one way ANOVA test)

- Non TZD users= Diabetic under metformin-sulfonylurea therapy. 


\section{DISCUSSION}

The insisting importance of therapy of diabetes mellitus with its disabling complications is emphasized by the fact that the disease constitutes a problem for a large number of persons and touches, to a greater or lesser degree, the practice of every physician regardless of his specialty.

Thiazolidinediones (TZDs) are class of oral hypoglycemic drugs that have been commonly prescribed throughout the world for the treatment of type 2 diabetes mellitus. The TZDs improve insulin resistance (a major pathophysiologic abnormality in Type 2 diabetes) and also have pleiotropic effects on insulin secretion, lipid and adipose tissue metabolism, body fat distribution and vascular endothelial function (Wannakamo, wt al., 2012) ${ }^{6}$.

Recent evidence from an epidemiological study and an exploratory clinical trial suggests that the use of TZDs is associated with a decrease in bone density in both postmenopausal women (Schwartz, et al., 2006; Grey, et al., 2007) and older men with type 2 diabetes (Yaturu, et al., 2007). In vitro and in vivo studies demonstrated that TZDs may produce this adverse effect through increased adipogenesis and decreased osteoblastogenesis (Wan, et al., 2007); decreased expression of insulin-like growth factor I which may contribute to decreased bone formation (Giustina, et al., 2008); promotion of osteoclast differentiation and bone resorption (Canalis, et al., 2007); and/or the insulin-sensitizing effect of TZDs reduces circulating insulin levels and therefore the insulin anabolic effect on the bone is reduced (Okazaki, et al., 1999).

The purpose of this study was to find out if the use of pioglitazone, an oral antidiabetic drug belonging to TZDs class of medications, has any adverse effects on BMD and serum levels of vitamin $\mathrm{D}$, calcium, and creatinine.

The purpose of this study was to find out if the use of pioglitazone, an oral antidiabetic drug belonging to TZDs class of medications, has any adverse effects on BMD and serum levels of vitamin D, calcium, and creatinine in diabetic patients in Jeddah, KSA. The obtained results with pioglitazone were compared with resulted from nondiabetic group and non-TZD users (metformin-sulfonylurea) group.

There was no significant effect on bone mineral density (BMD) in the three groups. Regarding BMD at the lumbar spine (L2-L4), and femoral neck, BMD measurements at the lumbar spine showed no significant differences between the three groups $(P=0.761)$ with a mean value of $0.91 \pm 0.12,0.95 \pm 0.12$ and $0.94 \pm 0.16$ for TZD users, other ant-diabetic users and the control groups respectively, as shown in Table 2. Similarly, the BMD at the right and left femoral neck also showed no statistical significance with a $\mathrm{P}$ value of $(P=0.959)$ and $(P=$ 0.768) respectively. Where the mean of the BMD was compared using the ANOVA, it showed $0.93 \pm 0.1,0.94 \pm 0.13$ and $0.93 \pm 0.15$ in the three groups, as shown in Table 2 . These results were in contrast to other studies that showed a decrease in BMD and increased risk of fractures in patients on TZD therapy (Wan, et al., 2007; Okazaki, et al., 1999; Thrailkill, et al., 2005). Some of this contravention may be explained by factors that are included within bone quality, while some may be related to the other non-skeletal factors. In terms of bone quality, there have been suggestions that there are racial differences in bone strength due to genetic, nutritional, lifestyle, and hormonal factors (Marc, 2007; Bhudhikanok, et al., 1996). The possible other non-skeletal factors include the small number of patients studied in the present work as well as the variation of the dose of the drug, and the duration of therapy. 
In compare with other study that showed increase in Vitamin D $(25(\mathrm{OH}) \mathrm{D})$ level in type two diabetic patients on thiazolidinediones (Zeina, 2010), this study showed no significant difference between the three groups in the level of serum vitamin D. It is well recognized that fat mass affects vitamin D status, and low levels of vitamin D are more commonly found in subjects with higher adiposity as well as in those with metabolic syndrome (Young, et al., 2009; Cheng, et al., 2010; Hyppo, et al., 2008). This is likely due to sequestration of most vitamin D, a fat-soluble vitamin, in adipose tissue (Heaney, et al., 2009; Kumer, et al., 2012). Although alteration in the metabolism of vitamin D in adipose tissue cannot be entirely ruled out, the difference in fat mass is less likely to affect the serum level of vitamin D in this study, since both the groups of study subjects were well matched for BMI.

\section{CONCLUSION}

This study has many limitations. Firstly, the sample size may not have enough statistical power to significantly demonstrate difference in BMD between the three groups the sample size was small because TZD was not prescribed a lot in Jeddah (Rosiglitazone Avandia FDA withdrawn from the market, Pioglitazone Actos has FDA warnings to cause bladder cancer) .secondly, although subjects were well matched for age and body weight, they haven't been matched for their co-morbidities and current used medications other than anti-diabetics. Moreover, Medications for diabetes were not controlled and there was an imbalance in diabetic medications used between the three groups. In particular, sulfonylureas were used more often in the non TZD group. However, it is less likely that this would affect our results since sulfonylureas, despite its long history of use in the management of diabetes, have not been associated with decreased BMD. Lastly, we did not document the period of diabetes in patients and the period of anti-diabetic drug use. But we considered the use of anti-diabetics for not less than six months. Still, unknown duration of diabetes can affect the results since increasing evidence suggests that patients with type 1 or type 2 diabetes are at increasing risk of osteoporotic fractures.

In summary, we concluded that there is no difference in BMD between patients on TZD as compared to those on other anti-diabetic agents. Further cohort prospective studies with large samples are needed for more demonstration of the effect of different anti-diabetic drugs on bone health.

\section{REFEREANCES}

Alikiani M, Alikhani Z, Boyd C, et al. (2007): Advanced glycetion end product stimulate osteoblast apoptosis via the MAP kinase and cytosolic apoptotic pathways. BONE.;40:345-353

Bhudhikanok G, Wang M, Eckert K, Matkin C, Marcus R (1996): Differences in bone mineral in Asian and Caucasian Americans may reflect differences in bone size. $\mathrm{J}$ bone Miner Res 11:1545-1556.

Brandi, Italy M. L, Bone health and diabetes.( 2010): Medicographia;32:364-369

Canalis E, Giustina A, Bilezikian JP (2007): Mechanisms of anabolic therapies for osteoporosis. N Engl J Med. 357:905-916.

Cheng S., Massaro J.M., Fox C.S., Larson M.G., Keyes M.J., McCabe E.L., Robins S.J., O’Donnell C.J., Hoffmann U., Jacques P.F., Booth S.L., Vasan R.S., Wolf M., Wang, T.J. (2010): Diabetes 59,242-248. 
Giustina A, Mazziotti G, Canalis E. (2008): Growth hormone, insulin-like growth factors, and the skeleton. Endocr Rev. 29:535-559.

Grey A, Bolland M, Gamble G, Wattie D, Horne A, Davidson J, Reid IR (2007): The peroxisome proliferator-activated receptorgamma agonist rosiglitazone decreases bone formation and bone mineral density in healthy postmenopausal women: a randomized, controlled trial. J Clin EndocrinolMetab 92:1305-1310.

Heaney R.P., Horst R.L., Cullen D.M., Armas L.A. (2009): J. Am. Coll.Nutr. 28, 252-256.

Hyppo"nen E., Boucher B.J., Berry D.J., Power C. (2008): Diabetes 57,298-305.

Kumer B., Sankar A., Sachan A., Katyarma D, Sarma K, (2012): Effect of oral hypoglycemic agents on bone metabolism in patients with type-2 diabetes, J Clin Sci Res, 1:83-93.

Marc $C$ (2007): Racial differences in bone strength. Trans Am Clin Climatol Assoc., 118:305315.

Monami. M, Cresci.B, Colombini. A, Pala. L, Balzi. D, Gori. F, Chiasserini.V, Marchionni.N, Rotella.C, Mannucci.E (2008): Bone Fracture and hypoglycemic treatment in type2 Diabetic Patient. Diabetic care;31:2.

Munoz- Torres M, Jodar E, Escobar-Jimenez F, et al. (1996): Bone mineral density measured by dual x-ray absorpitmetry in Spanish patients with insulin-dependent diabetes mellitus. Calcif Tissue Int.;58;316-319.

Okazaki R, Toriumi M, Fukumoto S, Miyamoto M, Fujita T, Tanaka K, Takeuchi Y (1999): Thiazolidinediones inhibit osteoclast- like cell formation and bone resorption in vitro. Endocrinology 140:5060-5065

Priya Shah and Sunder (2010): Pioglitazone: side effect and safety profile Mudaliar; 9:347354.

Schwartz AV, Sellmeyer DE, VittinghoffE, Palermo L, Lecka-Czernik B, Feingold KR, Strotmeyer ES, ResnickHE, Carbone L, Beamer BA, Park SW, Lane NE, Harris TB, Cummings SR (2006): Thiazolidinedione use and bone loss in older diabetic adults. J Clin Endocrino Metab 91:3349-3354.

Thrailkill KM, Lumpkin CK, Jr, BunnRC, Kemp SF, Fowlkes JL (2005): Is insulin an anabolic agent in bone? Dissecting the diabeticbone for clues. Am $J$ PhysiolEndocrinolMetab 289:735-745.

Wan Y, Chong LW, Evans RM (2007): PPAR-gamma regulates osteoclastogenesis in mice. Nat Med 13:1496-1503.

WannakamolSonsingh, DuangchitPanomvana et al (2012): Comparisons Of Effectiveness, Safety, And Pharmacokinetic Parameters Between Low And High Doses Of Pioglitazone In Type 2 Diabetic Patients. Int J Pharm 2(1): 8-14.

Yamagishi S, Nakamura K, Inoue H (2005): possible participation of advanced glycationend products in pathogenesis of osteoporosis in diabetic patient. Med Hypotheses.;65:1013-1015.

Yaturu S, Bryant B, Jain SK (2007): Thiazolidinedione treatment decreases bone mineral density in type 2 diabetic men. Diabetes Care 30:1574-1576.

Young K.A., Engelman C.D., Hairston K.G., Haffner S.M., Bryer-Ash M., Norris J.M (2009): J. Clin Endocrinol Metab 94: 3306-3313.

Zeina $A$ (2010): Thiazolidinedione use and the risk fractures in patients with type-2 diabetes mellitus. J Clin Endocrinol Metab, 95 (2):592-600. 


\section{تأثير عقار البيوجليتازون على سلامة عظام مرضى البول السكري في مدينة جده بالمملكة العربية السعودية.}

عبدالرحمن الاهدل - محمود عبدالمنعم ـ الاء الغامدي ـ ضحى العمودي ـ خلود المحمدي - رنا الهاشمي

من قسم الصيدلة السريرية ـ كلية الصيدلة - جامعة الملك عبدالعزيز - المملكة العربية السعودية

اجرى هذا البحث على عقار بيوجليتازون ( احد مشتقات مجموعة الثيازوليدينديون المستخدمة في علاج مرض البرات البول

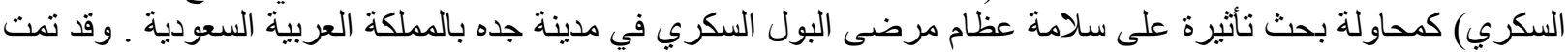

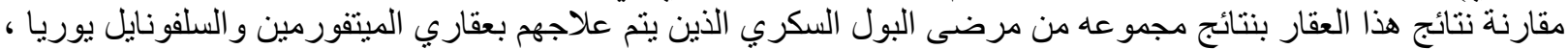

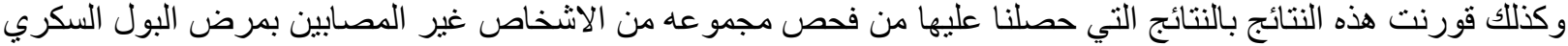

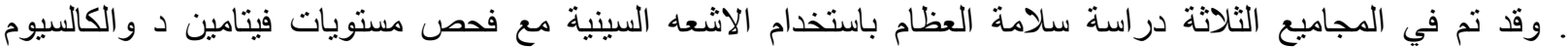

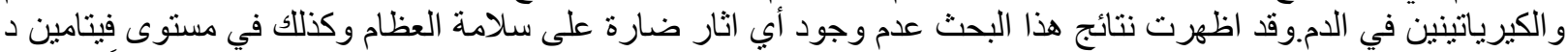

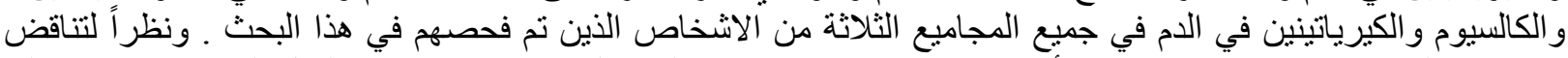

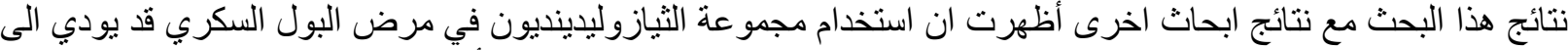

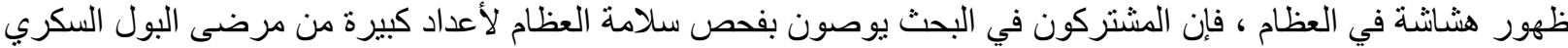

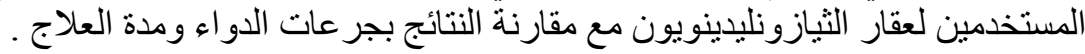

